\title{
Stellar vs. H II region chemical abundances in nearby galaxies
}

\author{
Fabio Bresolin \\ Institute for Astronomy \\ 2680 Woodlawn Drive, 96822 Honolulu, HI, USA \\ email: bresolin@ifa.hawaii.edu
}

\begin{abstract}
We have obtained new spectrophotometric data for $28 \mathrm{H}$ II regions in the spiral galaxy NGC 300, a member of the nearby Sculptor Group. The detection of several auroral lines has allowed us to measure electron temperatures and direct chemical abundances for the whole sample. We determine for the first time in this galaxy a radial gas-phase oxygen abundance gradient based solely on auroral lines. The gradient corresponds to $-0.077 \pm 0.006 \mathrm{dex} \mathrm{kpc}^{-1}$, which agrees very well with the galactocentric trend in metallicity obtained for $29 \mathrm{~B}$ and A supergiants in the same galaxy. The intercept of the regression for the nebular data virtually coincides with the intercept obtained from the stellar data. This result provides increased confidence on the direct method to determine extragalactic nebular abundances.
\end{abstract}

Keywords. HII regions, ISM: abundances, stars: abundances, galaxies: abundances

\section{Introduction}

The spectral analysis of $\mathrm{H}$ II regions has been an invaluable tool in astrophysics for the past few decades, providing a straightforward means to measure present-day chemical abundances in a variety of galactic environments, which has led, for example, to the study of radial abundance gradients in spiral galaxies (Vila-Costas \& Edmunds 1992, Zaritsky et al. 1994). It has recently become possible to extend the nebular techniques to star-forming galaxies at high redshift (Pettini et al. 2001), allowing the investigation of the cosmic chemical enrichment (Savaglio et al. 2005, Maier et al. 2006) in connection with fundamental properties of galaxies such as the mass-metallicity relation (Tremonti et al. 2004, Maiolino et al. 2008). In the local Universe, the knowledge of abundance gradients in spiral galaxies and their evolution with time provides the necessary observational constraints to the parameters that drive models of the chemical evolution of galaxies, such as the radial dependence of accretion and star formation rate in galactic disks (Matteucci \& Francois 1989, Chiappini et al. 2001).

The work on young massive stars to obtain reliable metallicities in nearby galaxies is less advanced, due to the need for a sophisticated NLTE treatment of the physical processes involving millions of metal lines in expanding atmospheres (Hillier \& Miller 1998, Pauldrach et al. 2001, Puls et al. 2005), and the requirement for telescopes with large collecting areas to secure spectra of individual stars located a few Mpc away (Bresolin et al. 2001, Bresolin et al. 2002). While it is possible to measure stellar metallicities from the integrated spectra of young star clusters (Larsen et al. 2006, 2008) or for starforming galaxies at high redshift (Rix et al. 2004, Halliday et al. 2008), more stringent tests that compare the chemical compositions of galaxies as obtained from $\mathrm{H}$ II regions and massive stars should be carried out in nearby, well-resolved systems. In doing so, the comparison is limited to chemical elements that are measurable in both types of objects and that, contrary to nitrogen, are largely unaffected by rotational mixing (Maeder 
\& Meynet 2000, Hunter et al. 2007). For these reasons the abundances of oxygen can be directly compared between ionized nebulae and young stars, mostly early-B dwarfs (within the Milky Way and in the Magellanic Clouds), and brighter A and B supergiants (in more distant galaxies). The expectation is that, once evolutionary effects in stars are properly accounted for, the present-day abundances derived from young massive stars and $\mathrm{H}$ II regions agree within the uncertainties of the measurements and of the modeling.

In low-metallicity and generally chemically homogeneous galaxies, such as the Magellanic Clouds and a small number of dwarf irregulars of the Local Group, the agreement found between H II region and young star chemical abundances is satisfactory (e.g. Trundle et al. 2005, Bresolin et al. 2006, Lee et al. 2006). The possibilities for comparison are even fewer in the case of spiral galaxies, as only data for the Milky Way (Rolleston et al. 2000, Deharveng et al. 2000, Daflon \& Cunha 2004) and M33 (Vilchez et al. 1988, Urbaneja et al. 2005a, Magrini et al. 2007, Rosolowsky \& Simon 2008) have insofar allowed meaningful comparisons. Yet, these are perhaps more interesting cases, because the metallicity in spiral galaxies can span a wide range, from the metal-rich nuclear regions to the metal-poor outskirts, of up to 1 dex (as in the case of M101, Kennicutt et al. 2003).

\section{Issues with nebular abundances}

Excluding the Milky Way and the Magellanic Clouds, H II regions often represent the only source of present-day abundance information for star-forming galaxies. However, despite their importance, nebular abundances are still subject to important systematic uncertainties. The classical, so-called direct method of measuring nebular abundances relies on the ability to measure the electron temperature $\left(T_{e}\right)$ of the gas, because the metal line strengths depend (via the line emissivity) exponentially on it. $T_{e}$ is obtained from the ratios of auroral (weak) to nebular (strong) lines, such as [O III $] \lambda 4363 / \lambda 5007$. In the vast majority of cases, however, extragalactic nebular abundances are derived from strongline metallicity indices, due to the difficulty of detecting the faint auroral lines, especially at high metallicity or at large redshift. These indices provide abundances of statistical value only, because they do not supply $T_{e}$ measurements. The main problem is that large differences (up to 0.7 dex!) can arise in the abundance values derived from strong-line methods, depending upon which calibration is adopted (Kewley \& Ellison 2008). Empirical calibrations that are tied to auroral line measurements (e.g. Pettini \& Pagel 2004) provide considerably smaller abundance values (0.2-0.6 dex) than theoretical calibrations, which are generated from grids of photoionization models (McGaugh 1991). This effect has been illustrated dramatically with the first direct measurements of electron temperatures in high-metallicity H II regions (Kennicutt et al. 2003, Bresolin et al. 2004). Given the importance of $\mathrm{H}$ II region abundances in disparate fields, from the distance scale (Bono et al. 2008) to the study of gamma-ray burst progenitors (Modjaz et al. 2008), it is essential to establish the accuracy of the methods used in measuring metallicities in star-forming galaxies.

\section{New nebular abundances in the spiral galaxy NGC 300}

Motivated by these considerations, recently we have analyzed new $\mathrm{H}$ II region spectra in the nearby spiral galaxy NGC 300 obtained with FORS2 at the VLT (Bresolin et al. 2009, Fig. 1). VLT spectra of $\sim 30$ blue supergiants in this galaxy had also been studied by our group (Urbaneja et al. 2005, Kudritzki et al. 2008), providing estimates of their metallicity, and thus offering the chance to compare stellar and nebular abundances. What makes such a comparison more significant than others is the fact that, instead of 


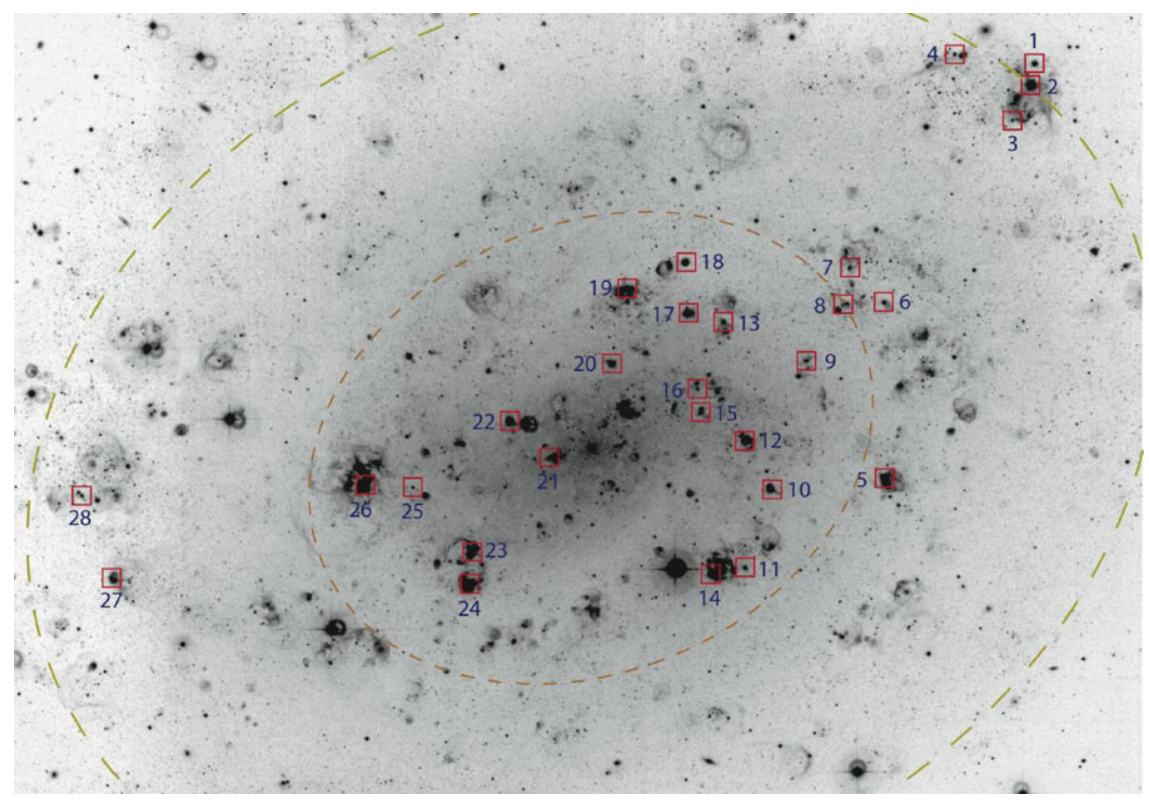

Figure 1. Location of the $\mathrm{H}$ II regions studied in this work on a narrow-band $\mathrm{H} \alpha$ image of NGC 300. The dashed lines represent the location of the projected $0.5 R_{25}$ and $R_{25}$ radii.

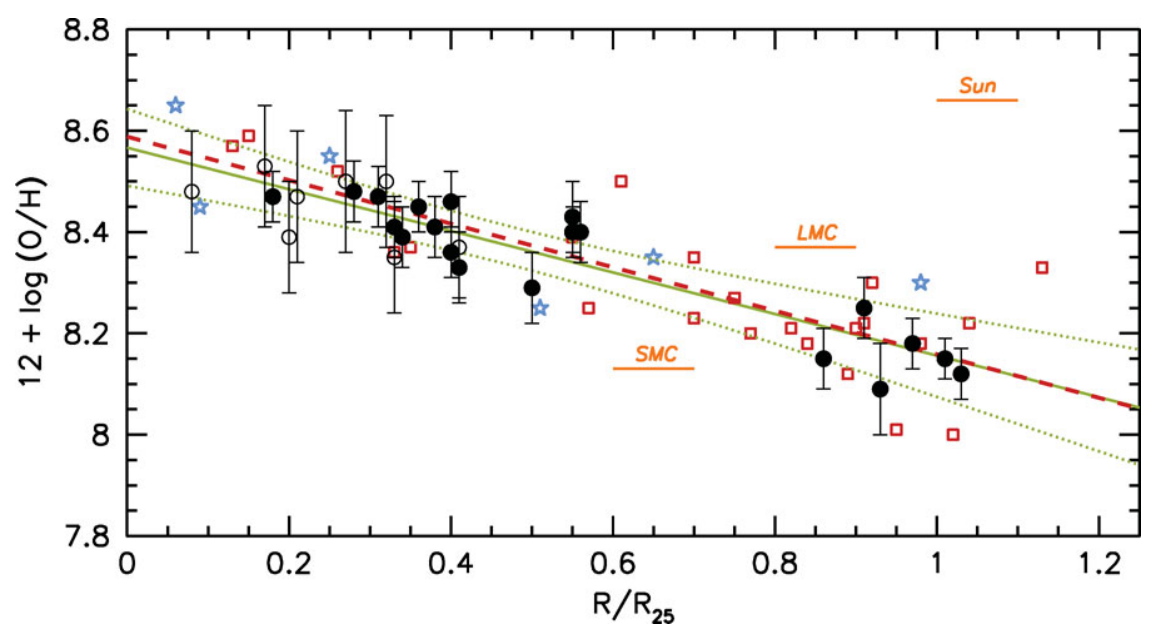

Figure 2. The radial oxygen abundance gradient in NGC 300 obtained from H II regions (circles) and blue supergiants (star symbols: B supergiants; open squares: A supergiants) by Bresolin et al. (2009), in terms of the isophotal radius $R_{25}$. The weighted regression line to the H II region data is shown by the continuous (green) line. The dotted lines show the $95 \%$ confidence level interval for the regression line. The dashed line represents the weighted regression to the BA supergiant star data. For reference, we include the oxygen abundances of the Magellanic Clouds and the solar photosphere. The central abundance of NGC 300, obtained from a linear extrapolation of the $\mathrm{H}$ II region data, is sub-solar, $12+\log (\mathrm{O} / \mathrm{H})=8.57 \pm 0.02$, and the slope of the radial abundance gradient is $-0.077 \pm 0.006 \mathrm{dex} \mathrm{kpc}^{-1}$. The metallicities of the $\mathrm{B}$ and $\mathrm{A}$ supergiants analyzed by us are fully consistent with the nebular results.

having to rely on gas-phase chemical abundances derived from strong-line methods (which are subject to the uncertainties mentioned above), we obtained a sample of $28 \mathrm{H}$ II regions for which we detected different auroral lines ([O III $] \lambda 4363$, [S III] $\lambda 6312$, [N II] $\lambda 5755$ ), which 
we used to derive electron temperatures and O, S, N, Ar and Ne abundances. This procedure yielded typical errors in $\mathrm{O} / \mathrm{H}$ of less than 0.1 dex. The main conclusion from our new work is that stellar and direct nebular abundances agree very well, in the range spanned by our objects, $12+\log (\mathrm{O} / \mathrm{H})$ approximately between 8.1 and 8.5 , i.e. from the metallicity of the Small Magellanic Clouds to an intermediate value between that of the Large Magellanic Cloud and the Sun (Fig. 2).

\section{References}

Bono, G., Caputo, F., Fiorentino, G., Marconi, M., \& Musella, I. 2008, ApJ, 684, 102

Bresolin, F., Kudritzki, R.-P., Mendez, R. H., \& Przybilla, N. 2001, ApJ, 548, L159

Bresolin, F., Kudritzki, R.-P., Najarro, F., Gieren, W., \& Pietrzynski, G. 2002, ApJ, 577, L107

Bresolin, F., Garnett, D. R., \& Kennicutt, R. C. 2004, ApJ, 615, 228

Bresolin, F., et al. 2006, ApJ, 648, 1007

Bresolin, F. et al. 2009, ApJ, 700, 309

Chiappini, C., Matteucci, F., \& Romano, D. 2001, ApJ, 554, 1044

Daflon, S. \& Cunha, K. 2004, ApJ, 617, 1115

Deharveng, L., et al. 1988, A\&AS, 73, 407

Halliday, C., et al. 2008, A\&SA, 479, 417

Hillier, D. J. \& Miller, D. L. 1998, ApJ, 496, 407

Kennicutt, R. C., Bresolin, F., \& Garnett, D. R. 2003 ApJ, 591, 801

Kewley, L. J. \& Ellison, S. L. 2008, ApJ, 681, 1183

Kudritzki, R.-P., et al. 2008, ApJ, 681, 269

Larsen, S. S., Origlia, L., Brodie, J., \& Gallagher, J. S. 2008, MNRAS, 383, 263

Larsen, S. S., Origlia, L., Brodie, J. P., \& Gallagher, J. S. 2006, MNRAS, 368, L10

Lee, H., Skillman, E. D., \& Venn, K. A. 2006, ApJ, 642, 813

Maeder, A. \& Meynet, G. 2000, ARA\&A, 38, 143

Magrini, L., Vilchez, J. M., Mampaso, A., Corradi, R. L. M., \& Leisy, P. 2007, A $\& A, 470,865$

Maier, C., et al.. 2006, ApJ, 639, 858

Maiolino, R., et al. 2008, A\& A, 488, 463

Matteucci, F. \& Francois, P. 1989, MNRAS, 239, 885

McGaugh, S. S. 1991, ApJ, 380, 140

Modjaz, M., et al. 2008, AJ, 135, 1136

Pauldrach, A. W. A., Hoffmann, T. L., \& Lennon, M. 2001, A\& A, 375, 161

Pettini, M., et al. 2001, ApJ, 554, 981

Pettini, M. \& Pagel, B. E. J. 2004, MNRAS, 348, L59

Puls, J., et al. 2005, A\& A, 435, 669

Rix, S. A., et al. 2004, ApJ, 615, 98

Rolleston, W. R. J., Smartt, S. J., Dufton, P. L., \& Ryans, R. S. I. 2000, A\&A, 363, 537

Rosolowsky, E. \& Simon, J. D. 2008, ApJ, 675, 1213

Savaglio, S., et al. 2005, ApJ, 635, 260

Tremonti, C. A., et al. 2004, ApJ, 613, 898

Trundle, C. \& Lennon, D. J. 2005, A\& $A, 434,677$

Urbaneja, M. A., et al. 2005a, ApJ, 635, 311

Urbaneja, M. A., et al. 2005b, ApJ, 622, 862

Vila-Costas, M. B. \& Edmunds, M. G. 1992, MNRAS, 259, 121

Vilchez, J. M., et al. 1988, MNRAS, 235, 633

Zaritsky, D., Kennicutt, Jr., R. C., \& Huchra, J. P. 1994, ApJ, 420, 87 